\title{
« Par delà les monts et dans le lointain » :l'armée britannique dans la péninsule ibérique (1808-1814)
}

\section{Charles Esdaile}

\section{(2) OpenEdition \\ 1 Journals}

\section{Édition électronique}

URL : https://journals.openedition.org/ahrf/1678

DOI : 10.4000/ahrf.1678

ISSN : 1952-403X

Éditeur :

Armand Colin, Société des études robespierristes

\section{Édition imprimée}

Date de publication : 1 juin 2004

Pagination : 53-70

ISSN : 0003-4436

\section{Référence électronique}

Charles Esdaile, « «Par delà les monts et dans le lointain » :l'armée britannique dans la péninsule ibérique (1808-1814) », Annales historiques de la Révolution française [En ligne], 336 | avril-juin 2004, mis en ligne le 15 juillet 2007, consulté le 23 avril 2022. URL : http://journals.openedition.org/ahrf/1678 ; DOI : https://doi.org/10.4000/ahrf.1678

Ce document a été généré automatiquement le 23 avril 2022.

Tous droits réservés 


\title{
«Par delà les monts et dans le lointain » :l'armée britannique dans la péninsule ibérique (1808-1814)
}

\author{
Charles Esdaile
}

« Au-delà des montagnes et des mers,

À travers les Flandres, le Portugal et l'Espagne,

La reine Anne commande et nous obéissons,

Par delà les monts et dans le lointain »

Comme le suggèrent les paroles de ce chant traditionnel des soldats du XVIIIe siècle, la péninsule ibérique a été un théâtre d'opérations bien connu de l'armée britannique bien avant la rencontre de Napoléon et de Wellington. Si l'Espagne et le Portugal n'étaient pas des destinations absolument habituelles pour les troupes britanniques en 1808, des incursions sur ces territoires témoignaient de la longue série de conflits qui avaient émaillé les relations franco-britanniques depuis le règne de Louis XIV. En 1704 avait débarqué en Espagne une expédition de 6500 hommes pour soutenir la cause des Habsbourg dans la Guerre de Succession, après quoi la présence britannique avait été maintenue au-delà de la défaite décisive de Villaviciosa en décembre 1710. Dans la guerre dite "oubliée » de 1718-1720, les forces anglaises avaient attaqué La Corogne, Vigo et Pontevedra. En juin 1762, 7000 soldats avaient été envoyés pour défendre le Portugal de l'invasion espagnole qui marqua les derniers jours de la guerre de Sept Ans. En 1800, des attaques amphibies avaient eu lieu à Cadix et El Ferrol. Et, en septembre 1806, la menace d'une nouvelle crise hispano-lusitanienne avait occasionné le départ d'une troisième expédition pour les rives du Tage, même si dans ce cas précis les troupes concernées ne foulèrent jamais le sol ibérique. En attendant, les Britanniques avaient maintenu une importante garnison à Gibraltar, qui avait soutenu un siège célèbre entre 1779 et 1783 , et depuis plusieurs années en avaient aussi installé une autre à Minorque. Bref, on voit que les soldats engagés au Portugal en août 1808 avaient une certaine familiarité avec le pays. Celle-ci n'était pas seulement faite de tuniques rouges et de mousquets de la Reine. Depuis des années un même fil conducteur a orienté les interprétations des guerres révolutionnaires et impériales. Pour résumer, 
tout le monde a affirmé que, pour défaire Napoléon, les puissances européennes durent utiliser les méthodes militaires qui avaient servi l'empereur comme avant lui la Révolution française. Comme l'écrit Blanning, « La France napoléonienne qui glissa vers une dictature militaire, c'était l'Ancien Régime qui se modernisait, mobilisait les milices citoyennes, déclarait la guerre totale et usait de la rhétorique de la libération $»^{1}$. Bien sûr, cette opinion est en fin de compte discutable, la réalité plus complexe. Cependant, même dans les cas où partie de l'avenir immédiat en dépendait, la réforme demeurait partielle et ne suscitait aucun enthousiasme car, dans le cas de la GrandeBretagne, de telles prétentions paraissaient simplement insensées. L'objet de cet article sera de démontrer combien la machine militaire échoua complètement à refléter les changements dans l'art de la guerre provoqués par la Révolution française et ses conséquences $^{2}$. Tant et si bien que le duc de Wellington aurait pu reconnaître l'armée de celui qui le précédait d'un siècle, le comte de Peterborough, sans la moindre difficulté, et vice versa. Selon les mots du spécialiste d'histoire militaire Hew Strachan, "L'armée britannique de 1815 semblait curieusement surannée »".

2 Si l'armée qui combattit à Waterloo relevait de l'ordre ancien, les raisons ne sont pas difficiles à comprendre. En bref, malgré l'ampleur de la menace représentée par la Révolution française et Napoléon, les Britanniques considéraient en fin de compte que leur art de la guerre traditionnel leur convenait parfaitement. Une marine omnipotente et efficace contrôlait les mers, protégeait les îles de l'invasion, tout en favorisant les raids sur les côtes ennemies et la conduite de grandes campagnes sur l'une ou l'autre région du continent, étendant les frontières de l'empire et établissant un monopole sur le commerce maritime en général et les richesses coloniales en particulier. Une milice puissante était en garnison dans le pays, maintenait la loi et l'ordre et surveillait les côtes. Une armée, relativement réduite mais aussi hautement professionnelle, apportait un appui en hommes aux alliés du continent menacés d'invasion - par exemple l'Espagne, le Portugal, la Sicile et la Suède - et secondait efficacement la diplomatie de la cour de St. James, prouvant que la Grande-Bretagne ne se contentait pas seulement, comme les cyniques l'ont parfois avancé, d'abattre le dernier Autrichien. Et la richesse engendrée par la plus grande puissance économique mondiale servait à acheter des mercenaires étrangers et pareillement des alliés continentaux. Il y avait certainement des différences par rapport aux affrontements initiaux - à partir de 1793, les traditionnelles milices des comtés avaient été amalgamées à des armées entières de volontaires patriotes mus (enfin, en théorie) par l'idéal d'une guerre idéologique contre la France, bien qu'une bien plus grande part du budget militaire passât aux troupes régulières. Mais fondamentalement c'était la même combinaison qui avait apporté la victoire à la Grande-Bretagne en 1714 et 1762 (ainsi la ferveur patriotique et la mobilisation massive suscitées par la peur de l'invasion n'étaient pas choses nouvelles : le XVIIIe siècle présente plusieurs cas de ces vagues d'enthousiasme populaire soulevées par la guerre, cependant que chaque nouveau conflit avait vu une augmentation de la proportion des effectifs britanniques sous les armes). Comme l'a remarqué Jeremy Black :

« Au XVIIIe siècle la Grande-Bretagne est généralement caractérisée par sa maîtrise des mers et son empire. L'image type des exploits de son armée est celle des hommes conduits par James Wolfe dans l'ascension des hauteurs d'Abraham, en 1759, pour préparer leur attaque sur Québec. C'est dans une large mesure une vue réaliste tant ce déploiement transocéanique était rempli de succès et l'utilisation des ressources militaires britanniques typique de l'histoire militaire contemporaine de la Grande-Bretagne. En outre, l'armée anglaise ne s'assortissait guère avec les 
concentrations massives d'un conflit continental à grande échelle, caractéristique des principales armées européennes (celle de France, celle de Grande-Bretagne) de la période. $»^{4}$

Passons maintenant à la machine militaire britannique. L'absence de réforme apparaitt un peu téméraire de la part de l'Angleterre face à la menace française. Cependant, si nous considérons la totalité des Britanniques sous les drapeaux dans la période 1803-1815, nous pouvons conclure que le pays s'est converti à la « nation en armes » à l'identique de la France. En additionnant l'armée régulière, la marine, les milices et les volontaires (la horde de régiments locaux formés à dessein de défendre la patrie, dont nous avons déjà parlé), en 1809786000 hommes, un sixième de la population adulte masculine, avait d'une façon ou de l'autre revêtu l'uniforme ; il a été de surcroît calculé que durant l'essentiel de la période les Anglais maintinrent une plus grande proportion de leurs forces mobilisées que ne le firent leurs adversaires français. Et, en théorie, deux décrets du Parlement - le « Levée en masse Act » de 1803 et le «Training Act » de 1806 - avaient introduit le principe du service obligatoire pour tous les hommes du pays. Mais les apparences sont trompeuses. En effet, plus de 500000 des 786000 hommes précités servaient soit dans les milices, soit dans les volontaires, avec pour conséquence qu'ils peuvent être pour partie décomptés (non seulement leur statut les limitait à la défense des îles de la métropole, mais leur discipline et l'état de leur préparation étaient si faibles qu'il était toujours indispensable de les épauler par d'importantes troupes régulières) ${ }^{5}$. En réalité, l'Angleterre faisait reposer sa guerre - ou ses campagnes - sur une armée régulière qui ressemblait beaucoup à celle du siècle précédent. S'il est vrai que les effectifs de celle-ci attinrent un niveau sans précédent durant les guerres napoléoniennes - 332000 hommes en 1813 au lieu des 132000 de 1803 -, cette croissance dut peu à l'enthousiasme populaire, alors que le système de recrutement n'était toujours pas modifié. Hormis les fruits limités de la brève tentative d'introduire une forme édulcorée de conscription en 1803-1804, les hommes de troupe relevaient d'un engagement volontaire, individuel ou issu de la milice (une source de recrutement à succès car les hommes qui s'engageaient étaient assurés de recevoir un degré d'instruction raisonnable et de surcroît de progresser dans leur vie de soldat). Pourtant, malgré l'exécration portée à Napoléon, l'armée ne parvint jamais à recruter suffisamment : entre juin et décembre 1803, période d'immense ferveur patriotique, les 360 recruteurs envoyés par le pays ne mobilisèrent que 3481 hommes, cependant qu'en 1811 l'on ne put compter que sur 865 volontaires de plus que le nombre de morts au combat. Cette situation tout à fait dramatique est récapitulée par Richard Holmes : "Durant les guerres napoléoniennes les pertes annuelles ne tombèrent jamais en dessous de 16071 hommes [...] et atteignirent 25498 en 1812. Les recrues en provenance des îles britanniques n'excédèrent jamais le nombre de 15308 (en 1808) et s'effondrèrent à 7367 en $1810 »^{6}$. Les conséquences pour l'armée furent terribles : la plupart des régiments d'infanterie ne purent maintenir qu'un bataillon sur le champ de bataille au lieu des deux réglementaires, tandis que l'encadrement de ces unités était réduit au minimum. En attendant, même le nombre limité de recrues qui se présentaient était fréquemment l'émanation de milieux peu recommandables : malgré l'offre d'une récompense financière substantielle - en 1805, un homme qui s'enrôlait dans l'infanterie gagnait environ 16 livres -, les seuls à se proposer volontiers étaient ceux qui l'avaient toujours fait. En bref, les seuls volontaires étaient des alcooliques chroniques, des apprentis en fuite, des criminels échappant à la loi, et d'autres réduits à la misère et au dénuement: d'où les milliers et les milliers de laboureurs irlandais qui 
grossirent les rangs de régiments tels que les Connaught Rangers et les Enniskillen Dragons, mais aussi ceux de nombreux régiments anglais et écossais, sans compter le grand nombre de tisserands artisanaux qui les rejoignirent après avoir vu leur mode de vie détruit par les progrès technologiques de l'industrie textile (n'oublions pas de mentionner aussi le Cent-unième Régiment, garnison permanente du sud du Pays de Galles, qui recrutait parmi les forçats en fin de peine de la colonie pénitentiaire de Botany Bay $)^{7}$. Pour le reste, les campagnes de recrutement recouraient à la fraude. Tant qu'il s'agissait de convaincre les vrais crédules, des promesses ridicules d'une vie facile et d'une solde élevée suffisaient. Mais dans tout autre cas, d'autres mesures s'imposaient et les recruteurs de chaque régiment trompaient leurs victimes infortunées à l'aide de la boisson afin de vaincre leur résistance ou leur faisaient croire au «shilling du roi ». Les mémoires de Benjamin Harris sont révélatrices de cet état de fait. Soldat au Quatre-vingt quinzième Régiment d'infanterie légère, Harris avait été envoyé comme agent recruteur dans le Sussex, à Hastings :

«En ces moments de recrutement, les responsables étaient habitués à se montrer sous leur meilleur jour et nous nous arrangeâmes trois fois mieux qu'à l'ordinaire. De ce fait le sergent-major était tout à fait pimpant ; il portait l'épée en bandoulière comme un officier supérieur, une sensationnelle plume verte à son chapeau, une écharpe flamboyante, [...] une pelisse d'officier sur ses épaules, et une double ration de rubans sur son couvre-chef, tandis que moi-même je m'étais fait aussi chic que j'osais apparaître, avec mon fusil sur le côté. Dans ces accoutrements nous fîmes du mieux que nous pûmes, comme si l'un et l'autre avions été généraux, et [...] ne fûmes pas sans faire sensation [...]. Nous remplîmes notre tâche avec ardeur. Je peux dire que durant trois jours ce fut une débauche de danse et de boisson. Chaque volontaire reçut une prime de dix guinées, qu'excepté les deux gardées pour le nécessaire, il dépensa en toutes sortes d'excès jusqu'à la dernière. $»^{8}$

4 À l'occasion, des moyens plus violents étaient employés. Dans la milice, par exemple, les hommes étaient fréquemment contraints par la menace à s'engager: on leur imposait des fatigues insoutenables ou de longues périodes de garde, tandis qu'en Écosse le vieux système clanique était exploité sans scrupules par des propriétaires terriens omnipotents tel Sir Thomas Graham, qui tentait d'assurer quantité de recrues pour le célèbre régiment des Highlands. On pouvait encore se procurer certaines d'entre elles en prononçant la traditionnelle condamnation des petits criminels à vivre sous la tunique rouge. Mais les pires de tous étaient les redoutés crimps, agents privés qui trompaient et éventuellement kidnappaient des individus insoupçonnables et ensuite les vendaient à l'armée ${ }^{9}$. Malgré tout, cela demeurait insuffisant : si la situation entre 1803 et 1815 présentait des améliorations par rapport à la précédente décade, lorsque les terribles pertes dues aux fièvres des Indes occidentales avaient sérieusement obéré le recrutement de l'armée régulière, les régiments recouraient de plus en plus aux campagnes propres à assurer celui-ci - de 360 à 1803 on passe à 1113 en 1807 - et étaient amenés à accepter pour grossir les rangs des hommes qui n'avaient ni l'âge ni la taille légaux, voire en mauvaise santé ${ }^{10}$.

5 Comment expliquer ce manque de recrues? Ce n'est pas comme si la pauvreté - à l'origine l'une des raisons majeures de s'engager - était absente: l'économie britannique non seulement traversait une période de graves difficultés durant les guerres contre la France, mais expérimentait aussi toutes les rigueurs de la révolution industrielle et agricole qui laissa beaucoup d'individus dans une situation des plus déplorables ${ }^{11}$. Nous devons donc trouver d'autres explications, non que ce soit particulièrement difficile. D'une part, l'armée pâtissait d'une réputation désastreuse 
dans l'opinion publique: ses soldats étaient, selon les mots célèbres du duc de Wellington, "la lie de la terre»; les récompenses en argent qui assuraient les enrôlements disparaissaient en général dans des coûts cachés (les recrues découvraient qu'il leur fallait payer uniformes et équipement et offrir la tournée à ceux qui les avaient choisies et à leurs nouveaux camarades); les conditions du service étaient terribles, même en temps de paix ; la discipline se caractérisait par l'usage fréquent du fouet, jusqu'à des condamnations à 1200 coups; l'avenir des vieux combattants était loin d'être rose quand bien même ils ne rentraient pas chez eux amputés d'un bras ou d'une jambe; en fin de compte, leur restait toujours offerte la chance d'une mort misérable dans une contrée éloignée. D'autre part, l'armée était en permanence en concurrence avec les milices et les volontaires, qui, d'une part, bénéficiaient de meilleures conditions de service et, d'autre part, n'exigeaient pas que leurs soldats servent à l'étranger. La plupart du temps, dans les zones côtières, c'était la Royal Navy que l'on sollicitait; la vie vraiment épouvantable des marins était contrebalancée par les primes les plus élevées et la promesse d'une part des richesses de chacun des bateaux pris à l'ennemi par l'équipage. Au cours de la guerre avec la France, diverses réformes furent entreprises afin de rendre moins décourageante la vie sous les drapeaux, la plus importante concernant la réduction du temps d'engagement - au lieu d'un contrat à vie - et le versement de pensions au terme de celui-ci. Dans le même temps, l'amélioration des manières et de la justice introduite par des officiers progressistes tel que Sir John Moore, et l'impressionnante kyrielle de victoires en Espagne et au Portugal, purent quelque peu changer la donne. Encouragées par les appels non déguisés au patriotisme, quelques rares personnes émergèrent qui ne se battaient plus seulement pour la recherche d'une gloire martiale mais étaient aussi avides de se battre pour leur pays. À leurs côtés on trouvait toujours quelques gentlemen du rang, riches de leur éducation et de leur savoir-vivre, qui avaient rejoint l'armée après quelque drame personnel ${ }^{12}$.Cependant, ceux qui, à partir de là, tentent de brosser une peinture idyllique des corps de troupe vont trop loin. Les Mémoires écrits longtemps après les événements ne doivent pas être pris pour argent comptant, d'autant que seule une minorité des engagés étant susceptible de s'adonner à la lecture et à l'écriture, son rôle est exagéré par rapport à son importance réelle. En réalité, les faits sont têtus : pour de bonnes raisons, on évitait et on méprisait le soldat lambda. Pour citer le duc de Wellington: "Les soldats anglais sont des individus qui se sont engagés pour boire. C'est une évidence : ils se sont tous engagés pour boire [...] Les gens disent que leur enrôlement relève d'une belle sensibilité au fait militaire: c'est n'importe quoi $»^{13}$. Et pour un témoignage venu du rang, citons les Mémoires de Joseph Donaldson, un Écossais venu sous les drapeaux pour échapper à sa vie fastidieuse d'employé :

«Il y avait peu de soldats [...] qui avaient quelque idée de la situation dans laquelle ils étaient. Ceux-là avaient peur de montrer qu'ils possédaient plus de connaissances que leurs camarades, peur d'être moqués par ceux qu'en d'autres circonstances ils auraient méprisés. Si un homme s'aventurait à parler dans un langage plus raffiné que celui de la société qui l'entourait, l'on disait que « Personne ne lisait le dictionnaire comme lui » ou "On ne nous a pas appris ces mots-là » ... S'il ne s'associait pas aux grivoiseries obscènes et imbéciles de ses voisins, il était taxé de "méthodiste»; s'il ne proférait pas insultes et jurons, il était un « quaker »; et s'il ne passait pas en boisson l'essentiel de sa paye, il était tenu pour un grippe-sou et un lâcheur et ses camarades l'exécraient [...] La voyouterie était bien portée et même les plus jeunes étaient prêts à s'abaisser à des scènes de débauche et de boisson. $»^{14}$ 
6 L'incapacité de l'armée à attirer un nombre suffisant de recrues était lourde de conséquences. En premier lieu, il était nécessaire de grossir les rangs de l'armée par un grand nombre de forains. Lors du conflit contre la France révolutionnaire, des régiments étrangers ont été créés en nombre considérable dont les dénominations de "Suisses » ou "Émigrants » cachaient une masse de déserteurs et de prisonniers de guerre de mauvaise réputation et d'une grande hétérogénéité. Avec la reprise des hostilités en 1803 , cette politique reprit de plus belle. Pensons au premier chef à la «King's German Legion », une force qui finit par compter jusqu'à dix bataillons d'infanterie, cinq régiments de cavalerie et cinq compagnies d'artilleurs. Fondée à l'origine sur des éléments de l'armée hanovrienne évacués vers la Grande-Bretagne en 1803, cette "Légion» fit toujours montre des preuves de patriotisme les plus manifestes, et l'on pouvait vanter la bravoure de ses soldats au combat. Pourtant, la réalité oblige à dire qu'ils étaient aussi recrutés dans les populations flottantes et les rebuts de la société. S'il y eut à la fin des fins un effort pour bel et bien limiter le recrutement aux seuls Allemands, un tel scrupule n'exista pas pour les sept autres régiments étrangers maintenus par les Britanniques entre 1803 et 1814, composés des hommes que l'on avait sous la main : Français, Allemands, Italiens, Suisses, Hollandais, Polonais, Croates et même Ukrainiens. Sans compter plusieurs régiments d'auxiliaires : neuf d'esclaves de l'Inde occidentale, huit de Canadiens, quatre de Ceylanais, deux issus de la colonie du Cap, un de Grecs, un de Corses et un de Siciliens. Il fallait y ajouter les régiments qui, bien qu'officiellement britanniques, recélaient un grand nombre d'étrangers. Le Soixantième, par exemple, mêlait Canadiens et Allemands, le Quatrevingt quinzième - le seul de l'armée de ligne entièrement armé de fusils en lieu et place des mousquets -, accueillait un certain nombre d'Espagnols; le Quatre-vingt dixseptième - les "Allemands de la Reine » - relevait d'une force de soldats autrichiens faits prisonniers à Minorque (déjà capturés par Napoléon lors des guerres d'Italie, ils avaient été recrutés par l'armée espagnole); et le Cent quatrième se résumait à l'origine à une unité de la milice canadienne. Conséquence : en 1813, il y avait au moins 53000 «tuniques rouges » - $50 \%$ des soldats de l'époque - qui n'étaient pas d'origine britannique ${ }^{15}$.

7 Malgré cette présence il n'y avait pas assez de soldats. L'État était obligé de garder des troupes régulières en nombre dans les îles britanniques pour les défendre d'une possible invasion française et aussi pour maintenir l'ordre public face à la combinaison $\mathrm{du}$ radicalisme politique et de la protestation sociale - en novembre 1811, 56000 hommes de l'armée régulière sont ainsi mobilisés. Comme de surcroît plusieurs régiments stationnaient partout sur le globe pour défendre les colonies anglaises, il était pratiquement impossible d'envoyer en Europe les forces nécessaires à des batailles comme celles d'Austerlitz, Iéna, Friedland ou Wagram. Pour surmonter ces difficultés, les gouvernements successifs devaient compter sur la disponibilité des forces étrangères. Ainsi, même à son plus haut, l'armée commandée en Espagne par Wellington n'excéda pas 60000 hommes, ce qui n'aurait pas permis de se maintenir sur le sol de la péninsule si les Espagnols - troupes régulières ou guérilla - n'avaient retenu l'attention de plusieurs milliers de soldats français. Tout aussi important pour Wellington, l'utilisation de l'armée portugaise pour compléter sa ligne de front - un stratagème identique sera employé à Waterloo, où les deux tiers des troupes qui défendront le Mont Saint-Jean seront des Allemands, des Hollandais et des Belges. Pour revenir à la guerre d'Espagne, la manière dont les Anglais fondent l'armée portugaise 
dans leur propre représentation nécessiterait un autre article, mais gardons à l'esprit qu'à partir de 1810 un tiers de l'armée de Wellington est constitué de Portugais, armés, entraînés et équipés de manière très voisine de leurs homologues espagnols, ce qui favorise une intégration totale (même si demeurent quelques formations portugaises indépendantes, la norme était d'amalgamer une brigade d'infanterie portugaise à deux brigades britanniques pour former une division; il est à noter que chaque bataillon portugais comptait plusieurs officiers britanniques, qui commandaient aussi les formations supérieures) ${ }^{16}$.

8 Ce qui résulte de tout cela, c'est une armée en campagne qui ne diffère guère des forces qui ont combattu sous l'Union Jack durant tout le dix-huitième siècle. Substituez les Hollandais aux Portugais et vous avez l'armée du duc de Marlborough; substituez les hommes de la Hesse aux Portugais et vous avez l'armée de Cornwallis. Et c'est particulièrement vrai si nous considérons les structures internes des armées. Un des principes fondamentaux de la nation en armes - le modèle que les opposants à Napoléon sont supposés avoir fait leur -, est que les carrières doivent s'ouvrir aux talents. De ce point de vue, il n'y avait depuis belle lurette quasiment plus d'obstacle à la démocratisation du corps des officiers britanniques. Pour un premier grade (comme enseigne dans l'infanterie, cornette dans la cavalerie), le postulant doit seulement savoir lire et écrire, obtenir une lettre d'approbation d'un supérieur du grade de colonel au moins et payer un droit qui variait de 400 à 1600 livres selon le régiment. La naissance, en bref, n'était pas un problème, les promotions pouvaient être monnayées ou prenaient en compte l'ancienneté, le mérite et les actes d'extrême bravoure sur les champs de bataille. Ce qui, toutefois, ne signifiait pas que les brillantes carrières d'officier étaient ouvertes à tous. Les jeunes gens de bonne famille mais aux ressources limitées pouvaient s'engager comme gentlemen volontaires avec l'espoir assuré d'obtenir une promotion sur le champ de bataille tandis que seul le simple soldat qui atteignait le statut de sergent pouvait être aussi sûr de son acceptation au mess des officiers. En bref, sans les moyens de son indépendance, il était difficile à quiconque de prospérer en tant qu'officier. Incapables de vivre de leur paye (le niveau de vie d'un officier était très coûteux), les particuliers sans le sou ne pouvaient soutenir les obligations d'un gentleman et il leur était par conséquent très difficile d'être acceptés par le reste des gradés et d'obtenir le respect de la troupe. Privés des possibilités de promotion, il leur restait à espérer de passer des années comme enseignes et lieutenants, d'autant qu'ils se trouvaient contraints d'accepter des postes peu enviés d'administrateurs qui les éloignaient du combat et de toute chance d'avancement pour acte de courage. Quoi d'étonnant à ce que beaucoup de ces anciens hommes du rang quittaient l'armée et acceptaient d'entrer dans les unités coloniales, au sein desquelles servir revenait à admettre une condamnation à mort virtuelle, ou bien sombraient dans l'alcool et la dépression? Tous les officiers ne furent pas gentlemen, même si leur naissance ne les désignait pas comme tels - aucun officier britannique n'eut jamais à exciper de ses quatre quartiers de noblesse comme c'était le cas dans la France de 1789 -, mais le goulot d'étranglement des privilèges était aussi resserré en 1815 qu'il l'était en $1792^{17}$.

9 À tout cela, les adversaires de la thèse selon laquelle l'armée britannique n'aurait changé ni de structure ni de nature depuis le XVIIIe siècle répondront que le fait que les officiers et les hommes fussent identiques ne les empêcha pas de trouver des moyens de lutter contre les Français qui n'avaient rien à voir avec les tactiques utilisées dans le siècle passé à Blenheim, Fontenoy ou Bunker Hill. Sans doute superficielle, cette 
assertion a quelque raison d'être du fait que dans les combats menés par Moore et Wellington l'infanterie légère joua un rôle important. Les troupes spécialisées, parfois armées de fusils, entraînées à se battre en ordre dispersé lors des escarmouches, nécessitaient un haut niveau d'initiative et de motivation individuelles (par contraste, les troupes qui combattaient en ordre serré pouvaient être facilement contrôlées par les officiers qui leur disaient que faire). Tandis que la plus grande partie de l'infanterie attendait sur deux lignes, les compagnies légères de chaque bataillon et les compagnies indépendantes de fusiliers rattachés à chaque brigade se déployaient en un éventail de plusieurs centaines de yards à l'avant des positions. Les généraux français, les voyant si nombreux, les confondaient parfois avec la ligne de bataille anglaise et ces professionnels de l'escarmouche harassaient sans pitié l'ennemi qui arrivait, enlevant les officiers, semant la confusion dans ses rangs et s'assurant que les Français ne parvinssent pas à s'approcher suffisamment pour endommager les troupes arrières en ordre rangé. Pour une description dramatique de cette méthode de combat, reportonsnous à nouveau aux mémoires de Benjamin Harris, qui fut l'un des acteurs de cette tactique lors de la bataille de Vimeiro, le 21 août 1808 :

«J'étais très vite tellement enveloppé par la fumée que je créais et le nuage qui qui planait autour de moi suite au feu continu de mes camarades que je ne pouvais rien voir sinon l'éclair rouge de mon arme. Les Français déferlaient sur nous en vagues continues et nous les arrosions de balles comme d'un shampoing de grêle de plomb. À couvert, nous tâtions le terrain, tirant un moment, grimpant et courant le suivant. $»^{18}$

$10 \mathrm{Vu}$ la part essentielle qu'elles prirent dans les constantes défaites subies par les Français qui affrontaient l'armée de Wellington en Espagne et au Portugal, l'existence de ces troupes suggère le développement d'un nouveau système tactique spécialement adapté aux manières de l'armée française. À tout le moins leur formation coïncida avec les guerres contre la France. Aussitôt que le conflit fut déclaré, des expériences commencèrent reposant sur l'utilisation de troupes légères sur le champ de bataille ; en 1794 apparaissaient les premiers régiments d'infanterie légère, des unités dans lesquelles les soldats étaient en théorie entraînés pour la guérilla comme la bataille en ordre rangé. De la même façon, le Soixantième Régiment Canadien fut doté en 1797 d'un cinquième bataillon entièrement armé avec le fusil Baker, qui, lors de la guerre d'Espagne, fut distribué dans des compagnies séparées dispersées dans différentes brigades d'infanterie selon les champs de bataille. En 1800, elles formèrent le fameux Quatre-vingt quinzième Régiment - qui, comme il a déjà été dit, était le seul entièrement équipé de fusils au lieu de mousquets -, qui devint immédiatement une des plus grandes unités de ligne anglaises, la seule à pouvoir aligner trois bataillons au lieu des deux ou un habituels. Finalement, sous l'influence de Sir John Moore, qui établit un camp d'entraînement spécial à Shorncliffe, sur la côte du Sussex, entre 1803 et 1809, six autres régiments à pied furent convertis en infanterie légère. D'autres unités légères furent éparpillées au sein de différentes légions étrangères, nous l'avons déjà précisé, ainsi que neuf bataillons de caçadores dans la nouvelle armée portugaise, preuve que les autorités britanniques accordaient une importance toute particulière à cette façon de faire la guerre ${ }^{19}$.

11 Cependant, il ne faut pas exagérer leur empressement quant à cette utilisation de l'infanterie légère. Les troupes spécialisées dans les escarmouches ne pouvaient à l'évidence changer seules la nature profonde de l'armée. Tout d'abord, la formation d'unités spéciales était dans les circonstances un pas en arrière. Sous l'influence de Sir 
John Moore, plusieurs de ces unités cultivaient un style différent de celui du reste de l'armée : l'usage du fouet disparaissait tandis qu'était encouragé l'esprit d'initiative et d'indépendance, avec des efforts particuliers pour stimuler le sens de l'honneur et du respect de soi. Mais si l'ampleur de ces changements était si grande qu'on le dit souvent, la création d'unités spéciales n'était pas une nouveauté, bien plus une réminiscence des pratiques communes du siècle écoulé - former des unités séparées pour s'adapter aux besoins nés des incursions, des escarmouches, des combats selon les difficultés du terrain. Elles étaient composées d'autre part de spécialistes hautement privilégiés formés dans des régiments permanents de fusiliers, paysans libres qui devaient le service militaire en temps de guerre en échange de leurs terres, ou criminels spécialement recrutés et volontaires attirés par les possibles pillages (voire, à l'occasion, bandes entières de bandits), toutes gens auxquels on pouvait faire confiance pour se battre seuls. Une telle solution était peut-être suffisante en un temps où la plupart des combats se faisaient en ordre fermé et où la plupart des soldats n'étaient pas entraînés à se battre individuellement, mais elle semblait désormais surannée car les Français ne comptaient pas, eux, sur des régiments spéciaux de troupes légères, mais demandaient plutôt à toutes leurs unités d'infanterie de déployer partie de leurs hommes pour les escarmouches. La vraie modernité résidait donc dans l'insertion au sein de chaque bataillon d'infanterie de ligne d'une compagnie légère entraînée à ce type de combat, mais telle avait été la pratique de l'armée depuis la guerre d'Indépendance américaine de 1776 à 1783, alors que la proportion des engagés était bien moindre que dans l'armée française (sur le tard, les bataillons d'infanterie disposèrent aussi de compagnies légères mais, comme ces bataillons comptaient six compagnies au lieu des dix habituels dans les forces britanniques, la proportion des spécialistes de l'escarmouche que les Français pouvaient jeter dans la bataille était nettement supérieure). Pour en revenir à la guerre d'Indépendance américaine, ce conflit avait montré que les Anglais avaient la capacité d'adapter leur tactique militaire aux besoins du moment: les lignes avaient été réduites à deux rangs au lieu de trois, tandis que les rabats et les queues de pie des uniformes avaient été coupés pour éviter aux hommes de s'enchevêtrer dans les épaisses broussailles qui couvraient l'essentiel du théâtre de la guerre. Ils s'étaient aussi débarrassés la plupart du temps du neck stock - une bande de cuir serrée autour du cou qui imposait au soldat de garder la nuque raide en permanence - et s'étaient dégagés de l'obligation de porter des cheveux gominés, poudrés et étirés en une insupportable queue de cheval, bien qu'aucun de ces changements n'ait été officialisé jusqu'à l'époque des guerres napoléoniennes. Ce n'était pas la seule fois durant le XVIIIe siècle que l'armée britannique avait répondu à de nouveaux impératifs. Lors de la guerre de Sept Ans, par exemple, le besoin de disposer d'un écran de cavalerie et d'améliorer la reconnaissance pour opérer dans les plaines d'Allemagne du Nord avait conduit à développer la cavalerie légère - les cavaliers montaient des coursiers à l'harnachement réduit, capables de porter rapidement les messages et d'être engagés dans des patrouilles sur de longues distances -, tandis que la révolte jacobite de 1745 avait montré la capacité tactique des soldats à s'adapter aux redoutables assauts des clans écossais. Tiraillée entre ses responsabilités aux Amériques et la nécessité de surveiller les confins sauvages des Highlands écossais, l'armée des années 1780 préparait une fois encore la confrontation continentale, comme en témoigne la fascination croissante exercée par les victoires de Frédéric le Grand, telle qu'elle transparaît dans la publication en 1792 des règlements de la nouvelle infanterie par Sir David Dundas. En bref, nous observons une instrument 
flexible toujours prêt à répondre aux besoins du moment, ce qui permet de replacer les changements observés durant la période napoléonienne dans leur vrai contexte, ne relevant pas d'une mutation fondamentale dans la nature de l'armée britannique ${ }^{20}$.

Le manque d'évolution dans la manière d'être de l'armée en général est souligné par la considération dont continue de jouir le système de récompenses et de punitions utilisé en dehors de l'infanterie légère. Le commun des soldats ne pouvait espérer aucune médaille d'aucune sorte jusqu'à la bataille de Waterloo comprise. Celui qui avait bien combattu n'avait pas davantage droit d'être cité, et ne recevait ni épée magnifiquement décorée ni mousquet d'exception spécialement manufacturé. De temps en temps un officier offrait à l'un de ces hommes une guinée d'or récompensant un acte d'héroïsme, tandis que quelques colonels progressistes instauraient un système de privilèges pour bonne conduite ou de gratifications sous forme d'insignes pour blessures et longs services. Cependant, cela demeurait exceptionnel. Si l'on fait abstraction du très mince espoir pour l'homme du rang d'obtenir une promotion, ne lui restait que la loyauté envers ses camarades et les couleurs. Ceci ne doit pas être sous-estimé car, pour beaucoup de "tuniques rouges ", leurs frères d'armes étaient leur seule famille et leur régiment leur seul foyer. Mais demeurait le problème de la peur. Dans quelques régiments de nouvelles méthodes de discipline furent appliquées à l'instar des évolutions constatées dans l'infanterie légère. Ainsi, dans telle unité, les délits mineurs étaient désormais punis de longues périodes de gardes durant lesquelles le coupable devait faire la sentinelle enchaîné à un tronc; dans telle autre, ceux qui avaient commis des fautes légères devaient passer plusieurs jours vêtus d'une blouse paysanne marquée d'une croix verte; dans une troisième, de telles canailles gardaient les latrines coiffés d'un bonnet de femme ${ }^{21}$. Mais de telles pratiques ne semblent pas avoir été très fréquentes. Même pour les délits les plus insignifiants, la punition habituelle était plutôt le fouet. Ainsi que nous l'avons déjà vu, le maximum des coups appliqués pour une même affaire était de 1200 coups, mais habituellement, les peines étaient de 300, 500 ou 700 coups. Il est fort probable que l'utilisation fréquente de la fustigation contribua à réduire le nombre des exécutions (selon Oman, seuls 118 soldats furent pendus ou fusillés au cours de la guerre d'Espagne). Néanmoins, cette pratique restait profondément choquante. Déshabillés jusqu'à la taille et attachés à un triangle de hallebardes, les punis étaient battus par un fouet connu sous le nom de « chat aux neuf queues", fait d'autant de lanières en cuir clouté longues d'au moins soixante centimètres, et leur dos était bientôt réduit à une masse de chair sanguinolente. Quelques-uns montraient un grand courage, mâchant des balles de mousquet ou des morceaux de cuir pour étouffer leurs cris mais, rapidement, la plupart s'évanouissaient, étaient ranimés et encore battus, à moins que la sentence ne soit achevée un autre jour. Pas étonnant que, lors de la guerre d'Indépendance américaine, les tuniques rouges se soient données le surnom de « dos en sang $»^{22}$.

La réforme partielle entreprise par l'armée britannique face à la menace napoléonienne peut être illustrée par deux autres thèmes. Tout d'abord, il est parfaitement clair que le déploiement d'un nombre important de spécialistes de l'escarmouche ne change pas la tactique de base de l'infanterie britannique, soit la bataille rangée. Les «light bobs", comme on les appelait, ne pouvaient arrêter une attaque française résolue, ayant plutôt pour mission de protéger l'arrière des troupes et de réduire l'impact de l'attaque ennemie. Bataille après bataille, les Français repoussèrent les assauts des « light bobs ", de manière à retrouver les conditions d'un combat plus familier à un général du XVIIIe siècle, contre des lignes d'infanterie anglaise alignées épaule contre épaule, sur deux 
rangs, et chacune soutenue le plus souvent par des batteries d'artillerie éparpillées (les armes anglaises n'étant pas en nombre suffisant et plus légères que celles des Français, leurs propriétaires ne pouvaient jamais égaler les imposantes masses d'artillerie alignées par les armées napoléoniennes, et de ce fait avaient tendance à utiliser leurs batteries seulement en soutien). Ce qu'il advenait ensuite est discutable, mais la vieille théorie -l'idée que l'infanterie britannique fauchait ses adversaires par des tirs répétés - paraît avoir du plomb dans l'aile. Défendue en 1910 dans un célèbre article de Sir Charles Oman, elle a été complètement battue en brèche par un récent travail qui a clairement montré que la tactique favorite des «tuniques rouges » était plutôt de tirer une seule volée, de se donner de sacrés encouragements et de partir à l'assaut baïonnette au canon, avec pour résultat invariable de faire fuir les Français en désordre ${ }^{23}$. Dans les campagnes du XVIIIe siècle une importance plus grande avait été accordée à la ligne de feu - de ce point de vue, il est important de noter que les nouveaux règlements de l'infanterie introduits en 1791 prohibent tout autre utilisation - mais la tactique utilisée dans la période napoléonienne, que l'habileté de Wellington sur le terrain, notamment s'il était en pente, rendra plus efficace, ne peut être citée en exemple d'une position progressiste à l'égard de la guerre de mouvement. Si cela correspondait en premier lieu à une forte compréhension de la psychologie de la bataille, des considérations pratiques s'imposaient aussi, tel que le fait bien connu qu'un feu continu déstabilisait une unité, semait la confusion dans ses propres rangs, l'enveloppant d'un nuage de fumée, rendant la plupart du temps impossible la transmission des ordres et ruinant toute possibilité d'avancer ou de manœuvrer. En fait, il n'y avait pas de période spécifique à ces phénomènes: la combinaison d'escarmouches et de combats en ligne était apparue durant la guerre d'Indépendance américaine tandis que la tactique de la volée unique, des cris et de la charge à la baïonnette était connue bien plus tôt, par exemple en 1743 lors de la bataille de Dettingen ${ }^{24}$.

Pour finir, portons nos regards vers les généraux anglais, et particulièrement vers le duc de Wellington. Là encore, nous observons davantage de continuité que de changement. Parmi d'autres, Sir John Moore, Sir Thomas Graham et Sir Rowland Hill peuvent tous être cités au titre de ces commandants d'une armée d'un nouveau style. Tous les trois étaient des hommes authentiquement aimables et respectables, opposés à l'usage du fouet et ardemment désireux de gagner l'affection de leurs soldats (Hill, de fait, était connu de ses hommes comme leur "Papa »). Mais beaucoup d'hommes de cet acabit servirent l'armée tout au long du XVIIIle siècle - le marquis de Granby, lord Cornwallis, Sir Ralph Abercromby et le grand duc d'York viennent à l'esprit - alors que la période napoléonienne compta aussi des cas comme ceux de Robert Craufurd et Thomas Picton, tous deux usant d'une discipline sévère qui relevait davantage de l'ancien ordre que du nouveau. Si nous nous intéressons à Wellington lui-même, nous trouvons là l'un des généraux qui marqua l'une des oppositions les plus irréductibles à la réforme - à toute réforme - dans toute l'histoire de l'armée britannique. Nous avons déjà évoqué son opinion du soldat britannique, mais il serait bon de rappeler une autre de ses remarques. Ainsi, écrit-il en 1813: "Il est impossible pour moi ou pour quiconque de diriger l'armée britannique dans le système actuel. Nous avons dans notre armée la lie de la terre comme simples soldats et dans les années récentes nous avons fait tout ce qui était en notre pouvoir pour relâcher la discipline qui est pourtant le seul moyen de gouverner de tels hommes ${ }^{25}$. Cette dureté, en vérité, était parfois adoucie - il faut noter qu'il parlait aussi des hommes du rang comme de «vieux 
compagnons » - mais au bout du compte courage et comportement impeccable étaient aux yeux de Wellington des produits de la sévérité, et de rien d'autre. Totalement opposé à l'école humanitaire qui s'interrogeait sur les égards dus aux soldats, Wellington avait aussi des doutes sérieux sur la possibilité d'en appeler à leur loyauté. Il n'en a usé d'aucune façon pour stimuler leur enthousiasme, persuadé que seuls comptaient le service et l'obéissance. Alors que les occasions ne manquèrent pas où ses troupes lui adressèrent des acclamations, il en manifesta un grand mécontentement, l'idée que le commun des soldats pût exprimer y compris une opinion favorable n'entrant pas dans ses schémas mentaux. La conséquence était un style de commandement réduit à sa plus simple expression: il n'eut jamais l'idée de visiter nuitamment le campement de ses hommes avant les batailles de Salamanque ou de Waterloo comme Napoléon l'avait fait avant Austerlitz, pas davantage de les stimuler sur le champ de bataille par des harangues sur le roi et la patrie ${ }^{26}$.

Nous sommes donc en présence d'une armée d'Ancien Régime, mue par des tactiques d'Ancien Régime, commandée par un général d'Ancien Régime. Ce qui devait être démontré l'a été: sans aucun doute, l'armée britannique était capable de relever le challenge de la Révolution française sans instiller des réformes fondamentales dans les forces armées ou la société. Composée de professionnels au long cours, l'armée de la Grande-Bretagne était entraînée à la manœuvre et aguerrie à une tactique aussi efficace en défense qu'en attaque. De surcroît elle avait l'avantage en Espagne d'être sous un général d'un talent incomparable qui s'était acquis la confiance massive de ses soldats. Comment être alors surpris qu'elle fût capable de surclasser l'armée impériale sans s'appuyer sur l'imposant programme de réformes mis en avant par les historiographes traditionnels des guerres napoléoniennes?

\section{NOTES}

1.T. Blanning, «La Révolution française et l'Europe », dans C. Lucas (éd.), Rewriting the French Revolution, Oxford, 1991, p. 206.

2.Pour discuter des limites de la réforme militaire chez les puissances orientales durant l'Empire, voir C. Esdaile, The Wars of Napoleon, Londres, 1995, pp. 186-215, 246-281, passim ; D. Gates, The Napoleonic Wars, Londres, 1997, pp. 226-231.

3.H. Strachan, « L'héritage de l'armée britannique au cours des guerres de la Révolution et de l'Empire ", dans J. Guy (éd.), The Road to Waterloo : the British Army and the Struggle against Revolution and Napoleonic France (1793-1815), Londres, 1990, p. 197.

4.J. Black, Britain as a Military Power (1688-1815), Londres, 1999, p. 45; voir aussi H.-V. Bowen, War and British Society (1688-1815), Cambridge, 1998, p. 7-19. Sur l'armée, en particulier, quelques très utiles points de comparaison peuvent être trouvés dans $\mathrm{R}$. Scouller, The Armies of Queen Anne, Oxford, 1996.

5.Pour les milices et les volontaires, voir I. Beckett, « La milice et les ennemis du roi (1793-1815) », dans Guy, op. cit., note 3, pp. 23-31 ; C. Emsley, « Le mouvement des volontaires ", ibid., pp. 40-47; et J. Cookson, « Le mouvement des volontaires durant les 
guerres napoléoniennes : du contexte ", Historical Journal, tome XXXII, $\mathrm{n}^{\circ} 4$, décembre 1989, pp. 867-892.

6.R. Holmes, Redcoat : the Britisch Soldier in the Age of tnhe Horse and Musket, Londres, 2001, p. 135.

7.Durant la période 1795-1810, $42 \%$ des recrues du Royal Artillerie étaient des Irlandais, en $180990 \%$ du Vingt-neuvième Régiment (comté de Worcester) et $34 \%$ du Cinquante-septième (Middlesex). Cf. Holmes, op. cit., note 6, pp. 55-56. À Waterloo, sur les 560 soldats de troupe appartenant au Vingt-troisième Régiment (Fusiliers de la Royal Welch), on ne comptait pas moins de cent tisserands désespérés. Cf. P.

Haythornthwaite, The Armies of Wellington, Londres, 1998, p. 49.

8.C. Hibbert (éd.), The Memoirs of Rifleman Harris, Londres, 1970, pp. 109-110. Pour en savoir plus sur le point de vue des recrues, voir C. O'Neil, The Military adventures of Charles O'Neil, Worcester (Massachussets, 1851), pp. 17-18.

9.Pour un exemple d'enlèvement pur et simple, voir A. Haley (éd.), The Soldier who walked away : autobiography of Andrew Pearson, a Peninsular-War Veteran, Liverpool, s.d., pp. 13-14.

10.Pour un aperçu général des méthodes de recrutement utilisées, voir $P$.

Haythornthwaite, Armies of Wellington, pp. 45-47, T. MC Guffie, « Recruter les rangs de l'armée régulière britannique pendant les guerres contre la France ", Journal of the Society of Army Historical Research, XXXIV, $n^{\circ} 138$ (juin 1956), pp. 50-58, et $n^{\circ} 139$ (septembre 1956), pp. 123-132.

11.Pour avoir idée de ces conditions, voir B. Coates, Wellington's Charge, Londres, 2002, pp. 91-95 et pp.115-118.

12.Pour avoir une idée des attentes nées des appels au patriotisme des masses, voir $\mathrm{L}$. James, Warrior Race : a History of the British at War, Londres, 2001, pp. 275-286; pour des vues nouvelles sur les soldats de ligne et du rang, voir Holmes, Redcoat, op. cit., pp. 139-151.

13.Comte de Stanhope, Notes of Conversations with the Duke of Wellington (1831-1851), Londres, 1889, pp. $14-18$.

14.J. Donaldson, Recollections in the Eventful Life of a Soldier, Edinburgh, 1852, pp. 44-45. 15.Concernant les étrangers servant la Grande-Bretagne, voir M. Yaple, «The auxiliaries : foreign and miscellaneous regiments in the British army (1802-1817) », Journal of the Society for Army Historical Research, tome L, n 201, printemps 1972, pp. 10-28.

16.Sur la réforme de l'armée portugaise et son assimilation aux forces de Wellington, voir F.-O. Cetre, « Beresford and the Portuguese army (1809-1814)», dans A. Berkeley (éd.), New Lights on the Peninsul War, Lisbonne, 1991, pp. 149-156.

17.Concernant le corps des officiers, voir C. Oman, Wellington's Army, Londres, 1913, p. 195-207 ; P. Haythornthwaite, Armies of Wellington, op. cit., pp. 22-34 ; M. Glover, «Purchase, patronage and promotion in the British army at the time of the Peninsular War », dans Army Quarterly and Defence Journal, tome CIII, $n^{\circ} 2$, janvier 1973, et $n^{\circ} 3$, avril 1973, pp. 355-362.

18. Hibbert, Recollections of Rifleman Harris, op. cit., pp. 26-27.

19.La plus importante étude sur le développement et l'utilisation de l'infanterie légère est celle de D. Gates, The British Light-Infantery Arm, 1790-1815 : its Creation, Training and Operational Role, Londres, 1987. Voir aussi A. Harman, « They decide not, nor are they chiefly relied on in battle », dans P. Griffith (éd.), A History of the Peninsular War, tome IX ("Modern Studies of the War in Spain and Portugal, 1808-1814"), Londres, 1999, pp. 
299-316. Sans doute serait-il bon de dire ici un mot du fusil Baker. Armant le cinquième bataillon du Soixantième régiment, le Quatre-vingt quinzième Régiment, les bataillons légers de la Légion allemande du Roi et quelques bataillons de caçadores, c'était une arme de grande qualité qui avait été inventée spécifiquement pour les combats de harcèlement. Son canon était coulé avec une spirale interne qui donnait un effet tournoyant aux balles lorsqu'elles étaient tirées tandis que le chargement - son contenu était enveloppé dans un cuir lubrifié - était réduit au minimum. Le tout donnait une arme dont le rayon d'action et l'exactitude dépassaient de beaucoup ceux des traditionnels mousquets. Elle pouvait également être chargée et utilisée en position couchée. Toutefois, le débit du tir restait lent - il fallait un petit maillet pour introduire les balles dans le canon - et la plupart des troupes continuèrent à favoriser le vieux «Brown Bess » (le mousquet de la reine).

20.Pour avoir une idée générale de la réforme militaire en Grande-Bretagne dans les années précédant la période napoléonienne, voir R. Glover, Peninsular Preparation : the Reform of the British Army, 1795-1809, Cambridge, 1963. Pour les mutations du XVIIIe siècle, cf. H. Rogers, The British Army of the Eighteenth Century, Londres, 1977, p. 44 et pp. 69-81.

21.P. Haythornthwaite, Armies of Wellington, op. cit., pp. 72-73.

22.Pour des témoignages contemporains sur les châtiments, l'un d'un officier, l'autre d'un homme du rang, voir G. Bell, Rough Notes of an Old Soldier, B. Stuart éd., Londres, 1956, p. 96-97, et G.-N. Bankes (éd.), The Autobiography of Seargeant William Lawrence, a Hero of the Peninsular War, Londres, 1886, pp. 48-49.

23. Ce débat peut être poursuivi par la lecture de C. Oman, Studies in the Napoleonic Wars, Londres, 1929, pp. 82-108, et de P. Griffith, Forward into Battle : Fighting Tactics from Waterloo to Vietnam, Chichester, 1981, pp. 12-43. Pour l'utilisation de l'artillerie par les Britanniques, cf. B. Hughes, Open Fire: Artillery Tactics from Malborough to Wellington, Chichester, 1983, pp. 62-74.

24.Sur les pratiques et l'expérience du XVIIIe siècle, voir C. Duffy, The Military Experience of the Age of Reason, Londres, 1987, pp. 204-214. Pour la bataille de Dettingen, dernière occasion où l'on vit un monarque anglais sur le champ de bataille, voir $\mathrm{J}$. Fortescue, A History of the British Army, Londres, 1910-1930, tome II, pp. 92-100.

25.Lettre de Wellington à Lord Bathurst, 2 juillet 1813. Université de Southampton, Papiers Wellington, I/373.

26.Pour avoir un exemple de l'hostilité de Wellington envers les acclamations de ses hommes, voir E. Longford, Wellington : the Years of the Sword, Londres, 1969, p. 560 ; en attendant, la plus fine analyse du caractère de Wellington demeure celle de C. Oman, A History of the Peninsular War, Oxford, 1902-1930, tome II, pp. 249-311.

\section{RÉSUMÉS}

Cet article discute la certitude des historiens de la période napoléonienne que l'Empire français fut seulement vaincu parce qu'il recourut aux armes de la Révolution française et, par-dessus tout, à la «nation en armes ». L'expérience britannique, était très différente. En effet, bien que 
jamais défaite sur le champ de bataille, l'armée du duc de Wellington en Espagne et au Portugal était le produit d'un système militaire entièrement fondé sur les pratiques du XVIIIe siècle. Reflet d'une stratégie qui évitait un engagement continental massif et préférait combiner guerres coloniales, blocus naval et alliances étendues, les troupes britanniques étaient peu nombreuses et recrutées selon des engagements volontaires parmi, majoritairement, les classes inférieures de la société. Le rang était grossi d'un grand nombre d'étrangers, que l'on utilisait tout aussi intensivement pour les auxiliaires (ce fut le cas au Portugal). La discipline était rude autant que la tactique paraissait d'un autre âge. Même si les Britanniques vainquirent quand même Napoléon en Espagne, les explications traditionnelles méritent d'être remises en question.

\section{Over the Hills and Far Away: the British army in the Peninsula, 1808-1814}

It is virtually an article of faith amongst historians of the Napoleonic era that the French empire was only defeated by recourse to the weapons of the French Revolution, and, above all, the Nation-in-Arms. The British experience, however, was very different. Thus, though never defeated in the field, the army commanded by the Duke of Wellington in Spain and Portugal was the product of a military system founded entirely in the practices of the eighteenth century. The reflection of a strategy that eschewed a massive continental commitment in favour of a combination of colonial warfare, naval blockade and great-power alliances, the British army was very small and recruited by voluntary enlistment from - in large part - the lowest classes of society. As in the eighteenth century, the ranks were swelled by large numbers of foreigners, whilst extensive use was also made of foreign auxiliaries (in this case the Portuguese). Discipline, too, was savage, whilst the army's tactical system was seemingly antiquated. Yet Britain still defeated Napoleon in the Peninsula, the traditional argument therefore being open to serious question.

\section{INDEX}

Mots-clés : Espagne, Guerre d'Espagne, armée britannique, duc de Wellington, vie militaire, guerres napoléoniennes, recrutement, tactique, stratégie

\section{AUTEUR}

\section{CHARLES ESDAILE}

University of Liverpool 